\author{
Miet Schetz \\ Michael Darmon
}

\section{Measuring acute kidney injury around the world: are we using the right thermometer (and adequately)?}

Received: 3 July 2015

Accepted: 6 July 2015

Published online: 14 July 2015

(C) Springer-Verlag Berlin Heidelberg and ESICM 2015

M. Schetz (『)

Clinical Department and Laboratory of Intensive Care Medicine, Division of Cellular and Molecular Medicine, KU Leuven University, Herestraat 49, 3000 Louvain, Belgium

e-mail: miet.schetz@scarlet.be

M. Darmon

Medical-Surgical Intensive Care Unit, Saint-Etienne University Hospital, Saint-Etienne, France

\section{Darmon}

Jacques Lisfranc Medical School, Jean Monnet University, Saint-Etienne, France

Acute kidney injury (AKI) is bad news in critically ill patients: it is associated with adverse short-term and longterm outcome and with important use of health care resources. Data on the incidence and outcome of AKI are essential to inform clinicians, scientists, and politicians. For decades epidemiological studies have been hampered by the absence of a consensus definition. During the last decade, three definitions of AKI have been developed: the RIFLE definition (Acute Dialysis Quality Initiative), the AKIN definition (Acute Kidney Injury Network), and finally the KDIGO definition (Kidney Disease Improving Global Outcomes) that reconciled the first two. These "modern" definitions and the associated staging criteria are based on increases of serum creatinine, decreases of urine output (UO), and need for renal replacement therapy (RRT) [1].
Several large observational studies, often based on retrospective analysis of existing databases, have addressed the epidemiology of AKI in critically ill patients using one of these "modern" definitions. The largest studies show highly variable results with an incidence ranging from 22 to $67 \%$ and mortality between 14 and $36 \%$ (Fig. 1). An independent association between AKI and mortality is nearly constantly demonstrated [2, $3]$.

Discrepancies between these epidemiological studies warranted a prospective worldwide evaluation of AKI in ICU patients using the most recent consensus definition. Hoste et al. undertook this difficult task and recently reported their results in Intensive Care Medicine [4]. Investigators were recruited at international ICU meetings. Between April 2009 and December 2010, 1802 patients from 97 centers in 33 countries were followed during their first ICU week. Three-quarters of the participating centers (treating $72 \%$ of the patients) were academic. AKI was diagnosed with the KDIGO definition/staging using both the creatinine and UO criteria and occurred in $57.3 \%$ of the patients. Baseline creatinine was known in $71 \%$ of the AKI patients; for those with unknown baseline the lowest of either the admission creatinine (17\%) or an MDRD (Modification of Diet in Renal Disease) back-calculated baseline $(11.7 \%)$ was used. Although rough incidence and mortality differed between continents and world zones, both adjusted incidence and mortality of AKI were similar across continents, world zones, country income, or proportion of the gross domestic product (GDP) spent on health care.

This study definitely provides interesting findings but also raises numerous questions. The most striking finding is the tremendously high incidence of AKI when UO criteria are taken into account and when the rate of missing baseline creatinine is reasonably low. Indeed, the incidence of AKI across studies may seem erratic. Beside usual factors affecting AKI incidence (case-mix, 


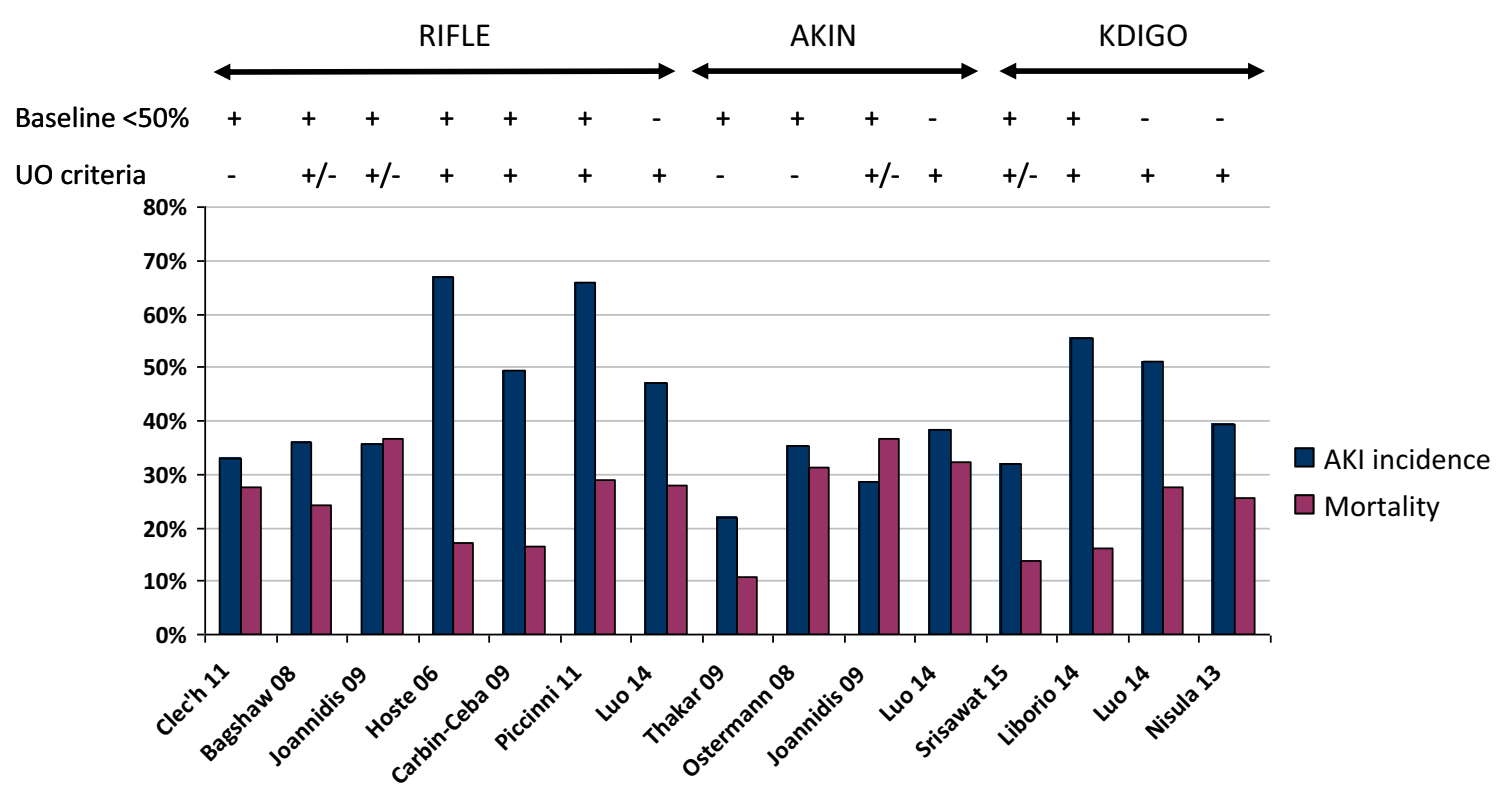

Fig. 1 Respective AKI incidence and mortality in large epidemiological studies according to definition, use of urine output criteria (UO), and rate of baseline creatinine effectively known (when opposed to estimated baseline creatinine; $<$ or $>50 \%$ of studied patients)

exclusion criteria, comorbidity, and illness severity), several factors directly related to the use of the modern definition may be influential. First, use of UO criteria has been shown to increase the sensitivity of the definition [5, 6] (Fig. 1). The optimal cut-off of these criteria remains debated, however, and their specificity is poor [7, 8]. Additionally, differences regarding the applied definition of baseline creatinine or the rate of unknown baseline creatinine greatly influence both AKI incidence and severity assessment (Fig. 1) [9]. The potential surrogates chosen to replace baseline creatinine have several limits. Use of admission serum creatinine, thus ignoring preadmission AKI, underestimates the true incidence of AKI [9]. Use of nadir serum creatinine overestimates the rate of AKI by overlooking muscle wasting during ICU stay [9, 10]. Last, use of MDRD-estimated baseline may result in both over- and underestimation of the incidence $[9,11$, 12] because this strategy assumes a low normal baseline glomerular filtration rate (thus ignoring pre-existing CKD) but also assumes that patients have comparable muscle mass as the age-, gender-, and weight-matched patients in the population used to derive the estimated glomerular filtration rate (eGFR) equation, which may not be the case in patients requiring ICU care. Thus, despite willingness to use consensual definitions, several limits should be kept in mind that may explain disparities across studies assessing AKI incidence.

This study has also several shortcomings. First, although the analysis provides an interesting snapshot of AKI in "worldwide" ICUs, the external validity of the results remains questionable. Africa and Asia are underrepresented and account for only 1.9 and $12.8 \%$ of included patients. None of the centers was in a low-income country, only $8.9 \%$ of the patients came from lower-middle-income countries, and only $5.9 \%$ were treated in countries where less than $5 \%$ of the GDP is spent on health care. These results are in line with a recent systematic review evaluating the world incidence of AKI [2]. In this systematic review, among the 3.6 million patients evaluated in 154 studies, the vast majority of the studies $(130 ; 84.4 \%)$ accounting for 3.3 million patients came from high-income countries and only two studies (2227 patients) originated from low- or low-middle-income countries [2]. Unlike Hoste et al., this study found mortality to be inversely correlated with proportion of GDP spent at health care and with the country gross national income per capita [2]. This discrepancy may have several explanations. First, the study by Hoste et al. may suffer from a selection bias since participating centers, recruited during international meetings, may not be representative for their country or continent [4]. Additionally, since most of the participating centers were academic, reported findings may illustrate differences in terms of AKI between large urban and rural areas [13]. The underrepresentation of low-middle-income countries may have contributed to lack of statistical power [4]. Finally, the adjusted analysis in Hoste et al. suggests that risk factors were most prevalent in countries with the highest incidence and mortality, which could reflect ICU admission policies in countries/centers with lower health care resources.

The study by Hoste et al. is informative, timely but also puzzling. The absent differences in adjusted incidence and mortality may be related to a lack of statistical power, 
might suggest that the process of care was similar over the world independent of resources, or that resources were similar in the participating centers. An alternative explanation is that the level of health care does not affect the incidence of AKI and that once AKI has occurred little can be done to change its prognosis. But this study also underlines that despite a decade of research using consensus definitions we are still struggling in assessing the incidence of AKI. This is partly due to the desire for definitions that are both useful for research and relevant for the clinical setting even though the lack of sensitivity and specificity of both creatinine and UO as markers of AKI preclude any adequate estimation of kidney injury $[7,14]$. In clinical practice other factors such as trends in kidney function, cause of AKI, comorbidity, and evolution of the underlying disease are mostly taken into account when managing AKI patients. In the research setting, it may perhaps be time to advance the field by accepting an imperfect but pragmatic definition, taking into account the frequent unavailability of baseline creatinine and the difficulties in estimating UO. In line with this, a European Renal Best Practice (ERBP) statement has already recommended the systematic use of a surrogate (first documented serum creatinine) as baseline creatinine and "shift based urine output" to homogenize patients' classification assuming that the KDIGO classification is a severity assessment rather than a nosological definition [15]. It would be dishonest to affirm that this recommendation is the only or optimal solution. But the idea is there: we need to move on.

\section{Compliance with Ethical Standards}

Conflicts of interest None.

\section{References}

1. Kellum JA, Lameire N, For the KDIGO AKI Guideline Work Group (2013) Diagnosis, evaluation, and management of acute kidney injury: a KDIGO summary (Part 1). Crit Care 17:204. doi:10.1186/cc11454

2. Susantitaphong P, Cruz DN, Cerda J et al (2013) World incidence of AKI: a meta-analysis. Clin J Am Soc Nephrol 8:1482-1493. doi: 10.2215/CJN.00710113

3. Nisula S, Kaukonen KM, Vaara ST et al (2013) Incidence, risk factors and 90 day mortality of patients with acute kidney injury in Finnish intensive care units: the FINNAKI study. Intensive Care Med 39:420-428. doi: 10.1007/s00134-012-2796-5

4. Hoste EAJ, Bagshaw SM, Bellomo R et al (2015) Epidemiology of acute kidney injury in critically ill patients: the multinational AKI-EPI study. Intensive Care Med. doi: 10.1007/s00134-015-3934-7

5. Wlodzimirow KA, Abu-Hanna A, Slabbekoorn M et al (2012) A comparison of RIFLE with and without urine output criteria for acute kidney injury in critically ill patients. Crit Care 16:R200. doi:10.1186/cc11808

6. Srisawat N, Sileanu FE, Murugan R et al (2015) Variation in risk and mortality of acute kidney injury in critically ill patients: a multicenter study. Am J Nephrol 41:81-88. doi: $10.1159 / 000371748$
7. Prowle JR, Liu YL, Licari E et al (2011) Oliguria as predictive biomarker of acute kidney injury in critically ill patients. Crit Care 15:R172. doi: 10.1186/cc10318

8. Legrand M, Jacquemod A, Gayat E et al (2015) Failure of renal biomarkers to predict worsening renal function in high-risk patients presenting with oliguria. Intensive Care Med 41:68-76. doi:10.1007/s00134-014-3566-3

9. Siew ED, Matheny ME, Ikizler TA et al (2010) Commonly used surrogates for baseline renal function can impact acute kidney Injury classification and prognosis. Kidney Int 77:536-542. doi: 10.1038/ki.2009.479

10. Schetz M, Gunst J, Van den Berghe G (2014) The impact of using estimated GFR versus creatinine clearance on the evaluation of recovery from acute kidney injury in the ICU. Intensive Care Med 40:1709-1717. doi: 10.1007/s00134-014-3487-1

11. Bagshaw SM, Uchino S, Cruz D et al (2009) A comparison of observed versus estimated baseline creatinine for determination of RIFLE class in patients with acute kidney injury. Nephrol Dial Transplant 24:2739-2744. doi:10.1093/ndt/gfp159

12. Závada J, Hoste E, Cartin-Ceba R et al (2010) A comparison of three methods to estimate baseline creatinine for RIFLE classification. Nephrol Dial Transplant 25:3911-3918. doi: $10.1093 / \mathrm{ndt} / \mathrm{gfp} 766$
13. Cerdá J, Bagga A, Kher V, Chakravarthi RM (2008) The contrasting characteristics of acute kidney injury in developed and developing countries. Nat Clin Pract Nephrol 4:138-153. doi: 10.1038/ncpneph0722

14. Waikar SS, Betensky RA, Bonventre JV (2009) Creatinine as the gold standard for kidney injury biomarker studies? Nephrol Dial Transplant 24:3263-3265. doi:10.1093/ndt/gfp428

15. Ad hoc working group of ERBP, Fliser D, Laville M et al (2012) A European Renal Best Practice (ERBP) position statement on the Kidney Disease Improving Global Outcomes (KDIGO) clinical practice guidelines on acute kidney injury: part 1: definitions, conservative management and contrastinduced nephropathy. Nephrol Dial Transplant 27:4263-4272. doi: 10.1093/ndt/gfs375 